Appl. Set-Valued Anal. Optim. 2 (2020), No. 3, pp. 339-349

Available online at http://asvao.biemdas.com

https://doi.org/10.23952/asvao.2.2020.3.08

\title{
STRONG CONVERGENCE OF THE VISCOSITY DOUGLAS-RACHFORD ALGORITHM FOR INCLUSION PROBLEMS
}

\author{
YAMIN WANG*, HAIXIA ZHANG \\ College of Mathematics and Information Science, Henan Normal University, Xinxiang 453007, China
}

\begin{abstract}
In recent years, the Douglas-Rachford algorithm received much attention due to its various applications in image recovery, signal processing, and machine learning. In this paper, we consider the Douglas-Rachford algorithm in the setting of Hilbert spaces. We introduce a viscosity Douglas-Rachford algorithm with multiparameters, and establish its strong convergence under some mild conditions.
\end{abstract}

Keywords. Douglas-Rachford algorithm; Hilbert space; Reflected resolvent; Strong convergence.

\section{INTRODUCTION}

In this paper, we assume that $H$ is a real Hilbert space with inner product $\langle\cdot, \cdot\rangle$ and induced norm $\|\cdot\|$. An important problem in the theory of nonlinear analysis is to find a solution of the following inclusion problem:

$$
0 \in(A+B) x
$$

where $A$ and $B$ are two monotone operators in Hilbert space $H$. In the paper, we denote by $z e r(A+B)$ the solution set of problem (1.1), and always assume that $z \operatorname{er}(A+B)$ is nonempty. Equation (1.1) includes many optimization problems arising from various applied areas such as signal processing, image recovery, statistical regression, and machine learning $[4,10,12,13,14]$ and the references therein.

Recently, various splitting algorithms have been investigated and weak-strong convergence theorems are obtained by many authors in the framework of different spaces; see, e.g., [5, 6, 11, $15,16]$ and the references therein. The Douglas-Rachford algorithm has become a very popular method to solve the sum problem (1.1). To numerically solve some types of heat equations, the Douglas-Rachford algorithm was first introduced by Douglas and Rachford [7] in 1956. In 1979, Lions and Mercier [11] extended the algorithm to be able to find a zero of the sum of two, not necessarily linear and possibly set-valued, maximally monotone operators. In 1992, Eckstein and Bertsekas [8] investigated the following Douglas-Rachford algorithm

$$
z_{k+1}=J_{\gamma A}\left(\left(2 J_{\gamma B}-I\right)\left(z_{k}\right)\right)+\left(I-J_{\gamma B}\right)\left(z_{k}\right),
$$

\footnotetext{
${ }^{*}$ Corresponding author.

E-mail addresses: wangyaminwangkai@163.com (Y. Wang), zhx6132004@ sina.com (H. Zhang)

Received August 27, 2020; Accepted November 30, 2020.
} 
where $\gamma>0$, and $J_{\gamma A}$ is the resolvent of $A$, which is defined as $J_{\gamma A}:=(I+\gamma A)^{-1}$. They proved that the above algorithm is a special case of the proximal point algorithm, and showed the weak convergence of the algorithm.

In 2004, Combettes [3] further proposed a relaxed extension of the Douglas-Rachford algorithm, and proved its weak convergence under some appropriate conditions.

In 2014, Bauschke and Noll [1] solved a feasibility problem with the aid of the DouglasRachford algorithm for two closed sets $A, B$ in a space $R^{d}$. Their Douglas-Rachford algorithm generated by

$$
x_{n+1} \in T\left(x_{n}\right), T:=\frac{1}{2}\left(R_{B} R_{A}+I d\right), n \in N,
$$

where $R_{A}=2 J_{A}-I d$ is reflected resolvent. Moreover, they proved that their algorithm converges to a fixed point of $T$ when $A, B$ are finite unions of convex sets.

In 2015, Bot, Csetnek and Hendrich [2] proposed an inertial Douglas-Rachford algorithm for finding the zeros of the sum of two maximally monotone operators in Hilbert spaces. Their algorithm reads as follows

$$
\left\{\begin{array}{l}
y_{n}=J_{\gamma B}\left[x_{n}+\alpha_{n}\left(x_{n}-x_{n-1}\right)\right], \\
z_{n}=J_{\gamma A}\left[2 y_{n}-x_{n}-\alpha_{n}\left(x_{n}-x_{n-1}\right)\right], \\
x_{n+1}=x_{n}+\alpha_{n}\left(x_{n}-x_{n-1}\right)+\lambda_{n}\left(z_{n}-y_{n}\right),
\end{array}\right.
$$

where $\gamma>0, x_{0}, x_{1}$ are arbitrarily chosen in $H,\left\{\alpha_{n}\right\}$ is a nondecreasing sequence with $\alpha_{1}=0$ and $0 \leq \alpha_{n} \leq \alpha<1$. They investigated its weak and strong convergence properties under some mild conditions.

In 2018, Dao and Phan [6] proposed a generalized Douglas-Rachford algorithm for feasibility problems. They established several local linear convergence results for the algorithm in solving feasibility problems with finitely many closed possibly nonconvex sets under different assumptions. The conclusions of their paper not only relaxed some regularity conditions but also improved linear convergence rates.

In 2019, Wang and Wang [15] proposed the following $\alpha$-Douglas-Rachford algorithm

$$
\left\{\begin{array}{l}
y_{n}=J_{B} x_{n} \\
z_{n}=J_{A}\left(\alpha y_{n}-x_{n}\right), \\
x_{n+1}=x_{n}+\left(z_{n}-y_{n}\right),
\end{array}\right.
$$

where $\alpha \in(1,2)$. Furthermore, they obtained the convergence of $\left\{x_{n}\right\},\left\{y_{n}\right\}$ and $\left\{z_{n}\right\}$ under some proper conditions in the framework of Euclidean spaces.

In this paper, motivated by the above results, we, based on a viscosity approximation technique, propose a viscosity Douglas-Rachford algorithm with multi-parameters to solve problem (1.1). We obtain its strong convergence under some mild conditions in the framework of Hilbert spaces.

\section{PRELIMINARY}

Let $A: H \rightarrow 2^{H}$ be a set valued operator. Recall that $A: H \rightarrow H$ is said to be monotone if

$$
\langle x-y, A x-A y\rangle \geq 0, \quad \forall x, y \in H .
$$


$A$ is said to be maximally monotone if it is monotone and its graph cannot be extended without destroying monotonicity. A mapping $T: H \rightarrow H$ is said to be nonexpansive if

$$
\|T x-T y\| \leq\|x-y\|, \quad \forall x, y \in H .
$$

Let $C$ be a nonempty closed convex subset of $H$. For any $x \in H$, there exists a unique vector, denoted by $P_{C} x$ in set $C$ with the property:

$$
\left\|x-P_{C} x\right\|=\min _{y \in C}\|x-y\| .
$$

$P_{C}$ is called the metric projection from $H$ onto $C$,

It is well-known that $P_{C} x$ is characterized by the following inequality:

$$
P_{C} x \in C, \quad\left\langle x-P_{C} x, z-P_{C} x\right\rangle \leq 0, \quad \forall z \in C .
$$

Definition 2.1. Let $T: H \rightarrow H$ be an operator. $T$ firmly nonexpansive if one of the following conditions is satisfied

(a) $2 T-I$ is nonexpansive;

(b) $\|T x-T y\|^{2} \leq\langle x-y, T x-T y\rangle$ for all $x, y \in H$;

(c) $\|T x-T y\|^{2} \leq\|x-y\|^{2}-\|(I-T) x-(I-T) y\|^{2}$ for all $x, y \in H$.

Definition 2.2. For a given number $v>0$, an operator $A: H \rightarrow H$ is said to be $v$-cocoercive (or $v$-inverse strongly monotone) if $\langle x-y, A x-A y\rangle \geq v\|A x-A y\|^{2}$ for all $x, y \in H$.

Next, we list some lemmas, which will be used for our main convergence theorem.

Lemma 2.1. [17] Let $\left\{s_{k}\right\}_{k=0}^{\infty}$ be a nonnegative real sequence such that

$$
s_{k+1} \leq\left(1-\lambda_{k}\right) s_{k}+\lambda_{k} b_{k}+c_{k},
$$

where $\left\{\lambda_{k}\right\},\left\{b_{k}\right\}$ and $\left\{c_{k}\right\}$ are real sequences satisfying the conditions:

(i) $\lim _{k \rightarrow \infty} \lambda_{k}=0, \sum_{k=0}^{\infty} \lambda_{k}=\infty$;

(ii) either $\limsup _{k \rightarrow \infty} b_{k} \leq 0$ or $\sum_{k=0}^{\infty}\left|\lambda_{k} b_{k}\right|<\infty$;

(iii) $c_{k} \geq 0$ for all $k$ and $\sum_{k=0}^{\infty} c_{k}<\infty$.

Then $\lim _{k \rightarrow \infty} s_{k}=0$.

Lemma 2.2. [9] Let $C$ be a nonempty closed convex subset of $H$ and let $T: C \rightarrow H$ be a nonexpansive mapping with Fix $(T) \neq \emptyset$. If $\left\{x_{n}\right\}$ is a sequence in $C$ such that $x_{n} \rightarrow x$ and $(I-T) x_{n} \rightarrow 0$, then $(I-T) x=0$, i.e., $x \in F i x(T)$.

Lemma 2.3. Let $A$ and $B$ be two monotone operators on a Hilbert space $H$. Let $x \in H$ and $\gamma>0$. Then

$$
x \in \operatorname{FixR}_{\gamma_{\mathrm{A}}} \mathrm{R}_{\gamma \mathrm{B}} \Leftrightarrow \mathrm{u}=\mathrm{J}_{\gamma_{\mathrm{B}} \mathrm{X}} \in \mathrm{S},
$$

where $R_{\gamma A}=2 J_{\gamma A}-I d$. 
Proof. Using the definition of the resolvent, we have

$$
\begin{aligned}
u=J_{\gamma B} x \in S & \Leftrightarrow 0 \in A\left(J_{\gamma B} x\right)+B\left(J_{\gamma B} x\right) \\
& \Leftrightarrow 0 \in \gamma A\left(J_{\gamma B} x\right)+\gamma B\left(J_{\gamma B} x\right) \\
& \Leftrightarrow J_{\gamma B} x \in \gamma A\left(J_{\gamma B} x\right)+(I+\gamma B)\left(J_{\gamma B} x\right) \\
& \Leftrightarrow J_{\gamma B} x \in \gamma A\left(J_{\gamma B} x\right)+x \\
& \Leftrightarrow 2 J_{\gamma B} x-x \in J_{\gamma B} x+\gamma A\left(J_{\gamma B} x\right) \\
& \Leftrightarrow 2 J_{\gamma B} x-x \in(I+\gamma A) J_{\gamma B} x \\
& \Leftrightarrow J_{\gamma A}\left(2 J_{\gamma B} x-x\right)=J_{\gamma B} x \\
& \Leftrightarrow 2 J_{\gamma A}\left(2 J_{\gamma B} x-x\right)-x=2 J_{\gamma B} x-x \\
& \Leftrightarrow\left(2 J_{\gamma A}-I\right)\left(2 J_{\gamma B}-I\right) x=x \\
& \Leftrightarrow x \in \operatorname{FixR}_{\gamma \mathrm{A}} \mathrm{R}_{\gamma B} .
\end{aligned}
$$

Lemma 2.4. Let $T=\left(2 J_{\gamma A}-I\right)\left(2 J_{\gamma B}-I\right)$, where $\gamma$ is any positive real number. Then $T$ is nonexpansive.

Proof. Since $J_{\gamma A}$ and $J_{\gamma B}$ are firmly nonexpansive, we have that $2 J_{\gamma A}-I$ and $2 J_{\gamma B}-I$ are nonexpansive according to Definition 2.1. For any $x, y \in H$, we have

$$
\begin{aligned}
\|T x-T y\| & =\left\|\left(2 J_{\gamma A}-I\right)\left(2 J_{\gamma B}-I\right) x-\left(2 J_{\gamma A}-I\right)\left(2 J_{\gamma B}-I\right) y\right\| \\
& \leq\left\|\left(2 J_{\gamma B}-I\right) x-\left(2 J_{\gamma B}-I\right) y\right\| \\
& \leq\|x-y\| .
\end{aligned}
$$

Hence, $T$ is nonexpansive.

The last lemma is trivial.

Lemma 2.5. In Hilbert spaces, we have

(i) $\|x+y\|^{2} \leq\|x\|^{2}+2\langle y, x+y\rangle$;

(ii) $\|\alpha x+(1-\alpha) y\|^{2}=\alpha\|x\|^{2}+(1-\alpha)\|y\|^{2}-\alpha(1-\alpha)\|x-y\|^{2}$;

(iii) $\|t x+s y\|^{2}=t(t+s)\|x\|^{2}+s(t+s)\|y\|^{2}-s t\|x-y\|^{2}$,

for any $x, y \in H$, and $\alpha, s, t \in R$.

\section{MAin Results}

In the section, we propose a viscosity Douglas-Rachford algorithm with multi-parameters for inclusion problem (1.1), and prove its strong convergence under some proper conditions.

Theorem 3.1. Let $A$ and $B$ be maximally monotone operators from $H$ to $2^{H}$ such that zer $(A+$ $B) \neq \emptyset$. Let $h: H \rightarrow H$ be a $\rho$-contraction for some $\rho \in[0,1)$. Let $\left\{\lambda_{n}\right\}$ be a sequence in $(0,2)$ such that

$$
0<c<\liminf _{n \rightarrow \infty} \lambda_{n} \leq \limsup _{n \rightarrow \infty} \lambda_{n}<d<2
$$


Let $\left\{\alpha_{n}\right\},\left\{\beta_{n}\right\},\left\{\delta_{n}\right\} \subseteq(0,1)$, and $\alpha_{n}+\beta_{n}+\delta_{n}=1$. For $\gamma>0$, and $x_{0} \in H$, set

$$
\left\{\begin{array}{l}
y_{n}=J_{\gamma B} x_{n} \\
z_{n}=J_{\gamma A}\left(2 y_{n}-x_{n}\right) \\
u_{n}=x_{n}+\lambda_{n}\left(z_{n}-y_{n}\right), \\
x_{n+1}=\alpha_{n} h\left(x_{n}\right)+\beta_{n} x_{n}+\delta_{n} u_{n} .
\end{array}\right.
$$

Suppose that the following conditions are satisfied

(i) $\lim _{n \rightarrow \infty} \alpha_{n}=0, \sum_{n=0}^{\infty} \alpha_{n}=\infty$;

(ii) $\lim _{n \rightarrow \infty} \frac{\alpha_{n}}{\delta_{n}}=0$.

Then there exists $z \in \Omega=F i x R_{\gamma A} R_{\gamma B}$ such that the following assertions hold:

(i) $\left\{x_{n}\right\}$ converges strongly to $z$, which is also the unique solution of the variational inequality:

$$
z \in \Omega,\langle(I-h) z, x-z\rangle \geq 0, x \in \Omega .
$$

Alternatively, $z$ is the unique fixed point of the contraction $P_{\Omega} h$, that is $z=P_{\Omega} h(z)$;

(ii) $\left\{y_{n}-z_{n}\right\}_{n \in N}$ converges strongly to 0 ;

(iii) $\left\{y_{n}\right\}_{n \in N}$ converges strongly to $J_{\gamma B} z$;

(iv) $\left\{z_{n}\right\}_{n \in N}$ converges strongly to $J_{\gamma B} z$.

Proof. Set $R_{\gamma A}=2 J_{\gamma A}-I$ and

$$
T=R_{\gamma A} R_{\gamma B}=\left(2 J_{\gamma A}-I\right)\left(2 J_{\gamma B}-I\right) .
$$

Moreover, as Lemma 2.3 indicates that $J_{\gamma B}(F i x T)=\operatorname{Zer}(A+B)$, which is nonempty, we have Fix $T \neq \emptyset$. From the definition of $u_{n}$, we can rewrite $u_{n}$ as

$$
\begin{aligned}
u_{n} & =x_{n}+\lambda_{n}\left[J_{\gamma A}\left(2 J_{\gamma B} x_{n}-x_{n}\right)-J_{\gamma B} x_{n}\right] \\
& =x_{n}+\lambda_{n}\left[2 J_{\gamma A} J_{\gamma B} x_{n}-J_{\gamma A} x_{n}-J_{\gamma B} x_{n}\right] \\
& =x_{n}+\lambda_{n} \frac{T-I}{2} x_{n} \\
& =\left(1-\frac{\lambda_{n}}{2}\right) x_{n}+\frac{\lambda_{n}}{2} T x_{n} .
\end{aligned}
$$

Setting $\rho_{n}=\frac{\lambda_{n}}{2}$, we have $0<\rho_{n}<1$. This together with (3.1) gives

$$
0<\frac{c}{2}<\liminf _{n \rightarrow \infty} \rho_{n} \leq \limsup _{n \rightarrow \infty} \rho_{n}<\frac{d}{2}<1 .
$$

Using (3.4), we have $u_{n}=\left(1-\rho_{n}\right) x_{n}+\rho_{n} T x_{n}$. Let $z \in F i x T=\Omega$ and $z=P_{\Omega} h(z)$, where $h(x)$ is a contraction with $\rho$. In view of Lemma 2.4 and Lemma 2.5 (ii), we obtain

$$
\begin{aligned}
\left\|u_{n}-z\right\|^{2} & =\left\|\left(1-\rho_{n}\right) x_{n}+\rho_{n} T x_{n}-z\right\|^{2} \\
& =\left(1-\rho_{n}\right)\left\|x_{n}-z\right\|^{2}+\rho_{n}\left\|T x_{n}-z\right\|^{2}-\rho_{n}\left(1-\rho_{n}\right)\left\|T x_{n}-x_{n}\right\|^{2} \\
& \leq\left(1-\rho_{n}\right)\left\|x_{n}-z\right\|^{2}+\rho_{n}\left\|x_{n}-z\right\|^{2}-\rho_{n}\left(1-\rho_{n}\right)\left\|T x_{n}-x_{n}\right\|^{2} \\
& =\left\|x_{n}-z\right\|^{2}-\rho_{n}\left(1-\rho_{n}\right)\left\|T x_{n}-x_{n}\right\|^{2} .
\end{aligned}
$$

Since $\rho_{n} \in(0,1)$, we can get that $\left\|u_{n}-z\right\| \leq\left\|x_{n}-z\right\|$. 
(i) To prove the convergence of $\left\{x_{n}\right\}$, we will divide our proof into four steps.

Step 1. Show that $\left\{x_{n}\right\}$ is a bounded sequence.

$$
\begin{aligned}
\left\|x_{n+1}-z\right\| & \leq \alpha_{n}\left\|h\left(x_{n}\right)-z\right\|+\beta_{n}\left\|x_{n}-z\right\|+\delta_{n}\left\|u_{n}-z\right\| \\
& =\alpha_{n}\left\|h\left(x_{n}\right)-h(z)+h(z)-z\right\|+\beta_{n}\left\|x_{n}-z\right\|+\delta_{n}\left\|u_{n}-z\right\| \\
& \leq \alpha_{n}\left\|h\left(x_{n}\right)-h(z)\right\|+\alpha_{n}\|h(z)-z\|+\beta_{n}\left\|x_{n}-z\right\|+\delta_{n}\left\|x_{n}-z\right\| \\
& \leq\left(\alpha_{n} \rho+\beta_{n}+\delta_{n}\right)\left\|x_{n}-z\right\|+\alpha_{n}\|h(z)-z\| \\
& =\left[1-\alpha_{n}(1-\rho)\right]\left\|x_{n}-z\right\|+\alpha_{n}(1-\rho) \frac{\|h(z)-z\|}{1-\rho} \\
& \leq \max \left\{\left\|x_{n}-z\right\|, \frac{\|h(z)-z\|}{1-\rho}\right\} \\
& \leq \max \left\{\left\|x_{0}-z\right\|, \frac{\|h(z)-z\|}{1-\rho}\right\} .
\end{aligned}
$$

Hence, $\left\{x_{n}\right\}$ is bounded.

Step 2. Show that the following inequality holds

$$
s_{n+1} \leq\left(1-\tilde{\alpha}_{n}\right) s_{n}+\tilde{\alpha}_{n} \omega_{n}
$$

where $s_{n}=\left\|x_{n}-z\right\|^{2}$,

$$
\tilde{\alpha}_{n}=\alpha_{n}\left(1-\rho^{2}\right)
$$

and

$$
\omega_{n}=\frac{2}{1-\rho^{2}}\left\langle h(z)-z, x_{n+1}-z\right\rangle-\frac{\delta_{n} \rho_{n}}{\alpha_{n}\left(1-\rho^{2}\right)\left(1-\alpha_{n}\right)}\left(1-\alpha_{n}-\delta_{n} \rho_{n}\right)\left\|T x_{n}-x_{n}\right\|^{2} .
$$

By Lemma 2.5 (i) and (ii), we can deduce

$$
\begin{aligned}
\left\|x_{n+1}-z\right\|^{2}= & \left\|\alpha_{n}\left(h\left(x_{n}\right)-z\right)+\beta_{n}\left(x_{n}-z\right)+\delta_{n}\left(u_{n}-z\right)\right\|^{2} \\
= & \left\|\alpha_{n}(h(z)-z)+\alpha_{n}\left(h\left(x_{n}\right)-h(z)\right)+\beta_{n}\left(x_{n}-z\right)+\delta_{n}\left(u_{n}-z\right)\right\|^{2} \\
\leq & \left\|\alpha_{n}\left(h\left(x_{n}\right)-h(z)\right)+\beta_{n}\left(x_{n}-z\right)+\delta_{n}\left(u_{n}-z\right)\right\|^{2} \\
& +2 \alpha_{n}\left\langle h(z)-z, x_{n+1}-z\right\rangle \\
= & \left\|\alpha_{n}\left(h\left(x_{n}\right)-h(z)\right)+\left(1-\alpha_{n}\right)\left[\frac{\beta_{n}}{1-\alpha_{n}}\left(x_{n}-z\right)+\frac{\delta_{n}}{1-\alpha_{n}}\left(u_{n}-z\right)\right]\right\|^{2} \\
& +2 \alpha_{n}\left\langle h(z)-z, x_{n+1}-z\right\rangle \\
\leq & \alpha_{n}\left\|h\left(x_{n}\right)-h(z)\right\|^{2}+\left(1-\alpha_{n}\right)\left\|\frac{\beta_{n}}{1-\alpha_{n}}\left(x_{n}-z\right)+\frac{\delta_{n}}{1-\alpha_{n}}\left(u_{n}-z\right)\right\|^{2} \\
& +2 \alpha_{n}\left\langle h(z)-z, x_{n+1}-z\right\rangle \\
\leq & \alpha_{n} \rho^{2}\left\|x_{n}-z\right\|^{2}+\frac{1}{1-\alpha_{n}}\left\|\beta_{n}\left(x_{n}-z\right)+\delta_{n}\left(u_{n}-z\right)\right\|^{2} \\
& +2 \alpha_{n}\left\langle h(z)-z, x_{n+1}-z\right\rangle .
\end{aligned}
$$


By Lemma 2.5 (iii) and inequality (3.6), we have

$$
\begin{aligned}
& \left\|\beta_{n}\left(x_{n}-z\right)+\delta_{n}\left(u_{n}-z\right)\right\|^{2} \\
= & \beta_{n}\left(\beta_{n}+\delta_{n}\right)\left\|x_{n}-z\right\|^{2}+\delta_{n}\left(\beta_{n}+\delta_{n}\right)\left\|u_{n}-z\right\|^{2}-\beta_{n} \delta_{n}\left\|u_{n}-x_{n}\right\|^{2} \\
\leq & \beta_{n}\left(1-\alpha_{n}\right)\left\|x_{n}-z\right\|^{2}+\delta_{n}\left(1-\alpha_{n}\right)\left[\left\|x_{n}-z\right\|^{2}-\rho_{n}\left(1-\rho_{n}\right)\left\|T x_{n}-x_{n}\right\|^{2}\right] \\
& -\beta_{n} \delta_{n}\left\|u_{n}-x_{n}\right\|^{2} \\
= & \left(1-\alpha_{n}\right)^{2}\left\|x_{n}-z\right\|^{2}-\delta_{n} \rho_{n}\left(1-\alpha_{n}\right)\left(1-\rho_{n}\right)\left\|T x_{n}-x_{n}\right\|^{2} \\
& -\beta_{n} \delta_{n}\left\|\left(1-\rho_{n}\right) x_{n}+\rho_{n} T x_{n}-x_{n}\right\|^{2} \\
= & \left(1-\alpha_{n}\right)^{2}\left\|x_{n}-z\right\|^{2}-\delta_{n} \rho_{n}\left(1-\alpha_{n}\right)\left(1-\rho_{n}\right)\left\|T x_{n}-x_{n}\right\|^{2}-\beta_{n} \delta_{n} \rho_{n}^{2}\left\|T x_{n}-x_{n}\right\|^{2} \\
= & \left(1-\alpha_{n}\right)^{2}\left\|x_{n}-z\right\|^{2}-\delta_{n} \rho_{n}\left[\left(1-\alpha_{n}\right)\left(1-\rho_{n}\right)+\beta_{n} \rho_{n}\right]\left\|T x_{n}-x_{n}\right\|^{2} \\
= & \left(1-\alpha_{n}\right)^{2}\left\|x_{n}-z\right\|^{2}-\delta_{n} \rho_{n}\left[\left(\beta_{n}+\delta_{n}\right)\left(1-\rho_{n}\right)+\beta_{n} \rho_{n}\right]\left\|T x_{n}-x_{n}\right\|^{2} \\
= & \left(1-\alpha_{n}\right)^{2}\left\|x_{n}-z\right\|^{2}-\delta_{n} \rho_{n}\left(\beta_{n}+\delta_{n}-\delta_{n} \rho_{n}\right)\left\|T x_{n}-x_{n}\right\|^{2} \\
= & \left(1-\alpha_{n}\right)^{2}\left\|x_{n}-z\right\|^{2}-\delta_{n} \rho_{n}\left(1-\alpha_{n}-\delta_{n} \rho_{n}\right)\left\|T x_{n}-x_{n}\right\|^{2} .
\end{aligned}
$$

It follows from (3.9) that

$$
\begin{aligned}
& \left\|x_{n+1}-z\right\|^{2} \\
\leq & \alpha_{n} \rho^{2}\left\|x_{n}-z\right\|^{2}+\frac{1}{1-\alpha_{n}}\left[\left(1-\alpha_{n}\right)^{2}\left\|x_{n}-z\right\|^{2}-\delta_{n} \rho_{n}\left(1-\alpha_{n}-\delta_{n} \rho_{n}\right)\left\|T x_{n}-x_{n}\right\|^{2}\right] \\
& +2 \alpha_{n}\left\langle h(z)-z, x_{n+1}-z\right\rangle \\
\leq & \alpha_{n} \rho^{2}\left\|x_{n}-z\right\|^{2}+\left(1-\alpha_{n}\right)\left\|x_{n}-z\right\|^{2}-\frac{\delta_{n} \rho_{n}}{1-\alpha_{n}}\left(1-\alpha_{n}-\delta_{n} \rho_{n}\right)\left\|T x_{n}-x_{n}\right\|^{2} \\
& +2 \alpha_{n}\left\langle h(z)-z, x_{n+1}-z\right\rangle \\
\leq & \left(1-\alpha_{n}\left(1-\rho^{2}\right)\right)\left\|x_{n}-z\right\|^{2}-\frac{\delta_{n} \rho_{n}}{1-\alpha_{n}}\left(1-\alpha_{n}-\delta_{n} \rho_{n}\right)\left\|T x_{n}-x_{n}\right\|^{2} \\
& +2 \alpha_{n}\left\langle h(z)-z, x_{n+1}-z\right\rangle \\
\leq & \left(1-\alpha_{n}\left(1-\rho^{2}\right)\right)\left\|x_{n}-z\right\|^{2}+\alpha_{n}\left(1-\rho^{2}\right)\left[\frac{2}{1-\rho^{2}}\left\langle h(z)-z, x_{n+1}-z\right\rangle\right. \\
& \left.-\frac{\delta_{n} \rho_{n}}{\alpha_{n}\left(1-\rho^{2}\right)\left(1-\alpha_{n}\right)}\left(1-\alpha_{n}-\delta_{n} \rho_{n}\right)\left\|T x_{n}-x_{n}\right\|^{2}\right] .
\end{aligned}
$$

Hence $s_{n+1} \leq\left(1-\tilde{\alpha}_{n}\right) s_{n}+\tilde{\alpha}_{n} \omega_{n}$ is valid.

Step 3. Show that $-\delta \leq \limsup _{n \rightarrow \infty} \omega_{n}<+\infty$ for some $\delta>0$, which indicates that $\limsup _{n \rightarrow \infty} \omega_{n}$ is finite. Since $\left\{x_{n}\right\}$ is bounded, we have that $\left\{\omega_{n}\right\}$ is bounded from above. In fact, we have

$$
\sup _{n \geq 0} \omega_{n} \leq \frac{2}{1-\rho^{2}}\|h(z)-z\|\left(\sup _{n \geq 0}\left\|x_{n+1}\right\|+\|z\|\right) .
$$


We next prove $\lim \sup \omega_{n} \geq-\delta$. To this aim, we proceed by contradiction. Assume that $\limsup \omega_{n}<-\delta$, which implies that there exists $n_{0} \in N$ such that $\omega_{n}<-\delta$ for all $n \geq n_{0}$. It $n \rightarrow \infty$
follows from that

$$
\begin{aligned}
s_{n+1} & \leq\left(1-\tilde{\alpha}_{n}\right) s_{n}+\tilde{\alpha}_{n} \omega_{n} \\
& \leq\left(1-\tilde{\alpha}_{n}\right) s_{n}-\tilde{\alpha}_{n} \delta \\
& =s_{n}-\tilde{\alpha}_{n}\left(s_{n}+\delta\right) \\
& \leq s_{n}-\left(1-\rho^{2}\right) \delta \alpha_{n} .
\end{aligned}
$$

By induction, we get

$$
s_{n+1} \leq s_{n_{0}}-\left(1-\rho^{2}\right) \delta \sum_{i=n_{0}}^{n} \alpha_{i} \text {, for all } n \geq n_{0}
$$

Hence by condition (i), $\limsup _{n \rightarrow \infty} s_{n}<s_{n_{0}}-\left(1-\rho^{2}\right) \delta \sum_{i=n_{0}}^{\infty} \alpha_{i}=-\infty$. As a matter of fact, $\left\{s_{n}\right\}$ is a nonnegative real sequence, which is a contradiction. Therefore, $\lim \sup \omega_{n}$ is finite.

Step 4. Prove that $\left\{x_{n}\right\}$ converges to $z=P_{\Omega} h(z)$. By Step 3, we can take a subsequence $\left\{\omega_{n_{k}}\right\}$ such that

$$
\begin{aligned}
\limsup _{n \rightarrow \infty} \omega_{n}= & \lim _{k \rightarrow \infty} \omega_{n_{k}} \\
= & \lim _{k \rightarrow \infty}\left(\frac{2}{1-\rho^{2}}\left\langle h(z)-z, x_{n_{k}+1}-z\right\rangle\right. \\
& \left.-\frac{\delta_{n_{k}} \rho_{n_{k}}}{\alpha_{n_{k}}\left(1-\rho^{2}\right)\left(1-\alpha_{n_{k}}\right)}\left(1-\alpha_{n_{k}}-\delta_{n_{k}} \rho_{n_{k}}\right)\left\|T x_{n_{k}}-x_{n_{k}}\right\|^{2}\right) .
\end{aligned}
$$

As $\left\{x_{n}\right\}$ is bounded, we have that $\left\{\left\langle h(z)-z, x_{n+1}-z\right\rangle\right\}$ is a bounded sequence of real number. Thus, without loss of generality, we may assume that the following limit exists

$$
\lim _{k \rightarrow \infty}\left\langle h(z)-z, x_{n_{k}+1}-z\right\rangle .
$$

Consequently, the following limit exists

$$
\lim _{k \rightarrow \infty} \frac{\delta_{n_{k}} \rho_{n_{k}}}{\alpha_{n_{k}}\left(1-\rho^{2}\right)\left(1-\alpha_{n_{k}}\right)}\left(1-\alpha_{n_{k}}-\delta_{n_{k}} \rho_{n_{k}}\right)\left\|T x_{n_{k}}-x_{n_{k}}\right\|^{2} .
$$

According to (3.5) and $\lim _{n \rightarrow \infty} \alpha_{n}=0$, we assume, without loss generality, that

$$
0<\frac{c}{2}<\rho_{n}<\frac{d}{2}<1,
$$

and $0<\alpha_{n}<1-\frac{d}{2}-\sigma$, where $0<\sigma<1-\frac{d}{2}$. Then

$$
\begin{aligned}
1-\alpha_{n_{k}}-\delta_{n_{k}} \rho_{n_{k}} & =\left(1-\delta_{n_{k}} \rho_{n_{k}}\right)-\alpha_{n_{k}} \\
& >1-\frac{d}{2}-\left(1-\frac{d}{2}-\sigma\right) \\
& =\sigma .
\end{aligned}
$$


It follows that

$$
\frac{\delta_{n_{k}} \rho_{n_{k}}}{\alpha_{n_{k}}\left(1-\rho^{2}\right)\left(1-\alpha_{n_{k}}\right)}\left(1-\alpha_{n_{k}}-\delta_{n_{k}} \rho_{n_{k}}\right)>\frac{\delta_{n_{k}} \rho_{n_{k}} \sigma}{\alpha_{n_{k}}\left(1-\rho^{2}\right)\left(1-\alpha_{n_{k}}\right)}>\frac{c \sigma}{2} \frac{\delta_{n_{k}}}{\alpha_{n_{k}}} .
$$

This together with (3.12) implies that the sequence $\frac{\delta_{n_{k}}}{\alpha_{n_{k}}}\left\|T x_{n_{k}}-x_{n_{k}}\right\|^{2}$ is bounded. Consequently, by condition (ii), we get

$$
\lim _{k \rightarrow \infty}\left\|T x_{n_{k}}-x_{n_{k}}\right\|^{2}=\lim _{k \rightarrow \infty} \frac{\alpha_{n_{k}}}{\delta_{n_{k}}}\left(\frac{\delta_{n_{k}}}{\alpha_{n_{k}}}\left\|T x_{n_{k}}-x_{n_{k}}\right\|^{2}\right)=0 .
$$

Lemma 2.2 guarantees that any weak cluster points of $\left\{x_{n_{k}}\right\}$ belongs to $\Omega$. By the definition of $x_{n_{k}+1}$, we deduce that

$$
\begin{aligned}
\left\|x_{n_{k}+1}-x_{n_{k}}\right\|= & \left\|\alpha_{n_{k}} h\left(x_{n_{k}}\right)+\beta_{n_{k}} x_{n_{k}}+\delta_{n_{k}} u_{n_{k}}-x_{n_{k}}\right\| \\
\leq & \alpha_{n_{k}}\left\|h\left(x_{n_{k}}\right)-h(z)+h(z)-z+z-x_{n_{k}}\right\|+\delta_{n_{k}}\left\|u_{n_{k}}-x_{n_{k}}\right\| \\
\leq & \alpha_{n_{k}}\left(\left\|h\left(x_{n_{k}}\right)-h(z)\right\|+\|h(z)-z\|+\left\|x_{n_{k}}-z\right\|\right) \\
& +\delta_{n_{k}} \rho_{n_{k}}\left\|T x_{n_{k}}-x_{n_{k}}\right\| \\
\leq & \alpha_{n_{k}}\left((1+\rho)\left\|x_{n_{k}}-z\right\|+\|h(z)-z\|\right)+\delta_{n_{k}} \rho_{n_{k}}\left\|T x_{n_{k}}-x_{n_{k}}\right\| \\
\leq & \alpha_{n_{k}} M+\left\|T x_{n_{k}}-x_{n_{k}}\right\| \rightarrow 0,
\end{aligned}
$$

where

$$
(1+\rho)\left\|x_{n_{k}}-z\right\|+\|h(z)-z\| \leq M .
$$

The last inequality is based on the fact that $\left\{x_{n}\right\}$ is a bounded sequence. This implies that any weak cluster points of $\left\{x_{n_{k}+1}\right\}$ also belongs to $\Omega$. Without loss of generality, we assume that $\left\{x_{n_{k}+1}\right\}$ weakly converges to $\bar{x}, \bar{x} \in \Omega$. Now by (3.11), we infer that

$$
\begin{aligned}
\limsup _{n \rightarrow \infty} \omega_{n} \leq & \lim _{k \rightarrow \infty} \frac{2}{1-\rho^{2}}\left\langle h(z)-z, x_{n_{k}+1}-z\right\rangle \\
= & \frac{2}{1-\rho^{2}}\langle h(z)-z, \bar{x}-z\rangle \\
& \leq 0
\end{aligned}
$$

which is due to the fact that $z=P_{\Omega} h(z)$ and the property of projections (2.1). Finally, from (3.7) we can apply Lemma 2.1 to deduce that $\left\|x_{n}-z\right\| \rightarrow 0$ as $n \rightarrow \infty$.

(ii) According to (i) and Lemma 2.4, for $z \in$ FixT, $\left\|x_{n}-z\right\| \rightarrow 0$,

$$
\begin{aligned}
\left\|x_{n}-T x_{n}\right\| & =\left\|x_{n}-z+z-T x_{n}\right\| \\
& =\left\|x_{n}-z+T z-T x_{n}\right\| \\
& \leq\left\|x_{n}-z\right\|+\left\|T x_{n}-T z\right\| \\
& \leq 2\left\|x_{n}-z\right\| \rightarrow 0
\end{aligned}
$$

as $n \rightarrow \infty$. From the definition of $\left\{y_{n}\right\}$ and $\left\{z_{n}\right\}$ and the above inequality, we have

$$
\begin{aligned}
\left\|y_{n}-z_{n}\right\| & =\left\|J_{\gamma A}\left(2 J_{\gamma B}-I\right) x_{n}-J_{\gamma B} x_{n}\right\| \\
& =\frac{1}{2}\left\|T x_{n}-x_{n}\right\| \rightarrow 0 .
\end{aligned}
$$


Therefore, (ii) is valid.

(iii) From the definition of $\left\{y_{n}\right\}$,

$$
\left\|y_{n}-J_{\gamma B} z\right\|=\left\|J_{\gamma B} x_{n}-J_{\gamma B} z\right\| \leq\left\|x_{n}-z\right\| \rightarrow 0
$$

as $n \rightarrow \infty$. Therefore, $\left\|y_{n}-J_{\gamma B} z\right\| \rightarrow 0$ as $n \rightarrow \infty$.

(iv) Combine (ii) and (iii), we can get $\left\{z_{n}\right\}$ converges strongly to $J_{\gamma B} z$ immediately.

Remark 3.1. Theorem 3.1 gives a viscosity Douglas-Rachford algorithm with multi-parameters, and shows its strong convergence. This will provide a new idea for the strong convergence of the Douglas-Rachford algorithm.

Corollary 3.1. Let $A$ and $B$ be maximally monotone operators from $H$ to $2^{H}$ such that zer $(A+$ $B) \neq \emptyset$. Let $\left\{\lambda_{n}\right\}$ be a sequence in $(0,2)$ such that

$$
0<c<\liminf _{n \rightarrow \infty} \lambda_{n} \leq \limsup _{n \rightarrow \infty} \lambda_{n}<d<2 .
$$

Let $\left\{\alpha_{n}\right\},\left\{\beta_{n}\right\},\left\{\delta_{n}\right\} \subseteq(0,1)$, and $\alpha_{n}+\beta_{n}+\delta_{n}=1$. For $\gamma>0$, and $x_{0}, u \in H$, set

$$
\left\{\begin{array}{l}
y_{n}=J_{\gamma B} x_{n}, \\
z_{n}=J_{\gamma A}\left(2 y_{n}-x_{n}\right), \\
u_{n}=x_{n}+\lambda_{n}\left(z_{n}-y_{n}\right), \\
x_{n+1}=\alpha_{n} u+\beta_{n} x_{n}+\delta_{n} u_{n} .
\end{array}\right.
$$

Suppose that the following conditions are satisfied

(i) $\lim _{n \rightarrow \infty} \alpha_{n}=0, \sum_{n=0}^{\infty} \alpha_{n}=\infty$;

(ii) $\lim _{n \rightarrow \infty} \frac{\alpha_{n}}{\delta_{n}}=0$.

Then there exists $z \in \Omega=F i x R_{\gamma A} R_{\gamma B}$ such that the following assertion hold:

(i) $\left\{x_{n}\right\}$ converges strongly to $z=P_{\Omega} u$;

(ii) $\left\{y_{n}-z_{n}\right\}_{n \in N}$ converges strongly to 0 ;

(iii) $\left\{y_{n}\right\}_{n \in N}$ converges strongly to $J_{\gamma B} z$;

(iv) $\left\{z_{n}\right\}_{n \in N}$ converges strongly to $J_{\gamma B} z$.

\section{Acknowledgments}

The research of Y. Wang was supported in part by the National Natural Science Foundation of China with grant no.11901171, and the Foundation of Henan Educational Committee with grant no.16A110017. The research of H. Zhang was supported in part by the National Natural Science Foundation of China with grant no.11701154.

\section{REFERENCES}

[1] H. H. Bauschke, D. Noll, On the local convergence of the Douglas-Rachford algorithm, Archiv der Math. 102 (2014), 589-600.

[2] R. I. Bot, E. R. Csetnek and C. Hendrich, Inertial Douglas-Rachford splitting for monotone inclusion problems, Appl. Math. Comput. 256 (2015), 472-487.

[3] P. L. Combettes, Solving monotone inclusions via compositions of nonexpansive averaged operators, Optimization, 53 (2004), 475-504. 
[4] T.H. Cuong, J.C. Yao, N.D. Yen, Qualitative properties of the minimum sum-of-squares clustering problem, Optimization, 69 (2020), 2131-2154.

[5] S.Y. Cho, X. Qin, L. Wang, Strong convergence of a splitting algorithm for treating monotone operators, Fixed Point Theory Appl. 2014 (2014), Article ID 94.

[6] M. N. Dao, H. M. Phan, Linear convergence of the generalized Douglas-Rachford algorithm for feasibility problems, J. Global Optim. 72 (2018), 443-474.

[7] J. Douglas, H. H. Rachford, On the numerical solution of the heat conduction problem in 2 and 3 space variables, Trans. Amer. Math. Soc. 82 (1956), 421-439.

[8] J. Eckstein, D. P. Bertsekas, On the Douglas-Rachford splitting method and the proximal point algorithm for maximal monotone operators, Math. Program. 55 (1992), 293-318.

[9] K. Goebel, W. A. Kirk, Topics on metric fixed point theory, Cambridge University Press, Cambridge, 1990.

[10] L.V. Nguyen, X. Qin, The minimal time function associated with a collection of sets, ESAIM Control Optim. Calc. Var. 26 (2020), 93.

[11] P. L. Lions, B. Mercier, Splitting algorithms for the sum of two nonlinear operators, SIAM J. Numer. Anal. 16 (1979), 964-979.

[12] X. Qin, N.T. An, Smoothing algorithms for computing the projection onto a Minkowski sum of convex sets, Comput. Optim. Appl. 74 (2019), 821-850.

[13] S. Sra, S. Nowozin, S. J. Wright, Optimization for machine learning, MIT Press, 2012.

[14] R. Tibshirani, Regression shrinkage and selection via the lasso, J. Royal Statist. Soc., Series B, 58 (1996), 267-288.

[15] D. Wang, X. Wang, A parameterized Douglas-Rachford algorithm, Comput. Optim. Appl. 73 (2019), 839869.

[16] W. Takahahsi, H.K. Xu, J.C. Yao, Iterative methods for generalized split feasibility problems in Hilbert spaces, Set-Valued Var. Anal. 23 (2015), 205-221.

[17] H. K. Xu, Iterative algorithms for nonlinear operators, J. London Math. Soc. 66 (2002), 240-256. 